\title{
Water in small bodies of the Solar System
}

\section{Dominique Bockelee-Morvan}

Observatoire de Paris, Meudon, France

email: dominique.bockelee@obspm.fr

\begin{abstract}
Water in form of ice or vapour is observed in comets, transneptunian objects and icy satellites formed in the outer regions of the Solar System, as well as in objects orbiting in the inner Solar System, such as dwarf planet Ceres. I will present an overview of the water content and properties in these objects and the implications in terms of solar system formation and evolution.
\end{abstract}

Keywords. 